Pacific Journal of Mathematics

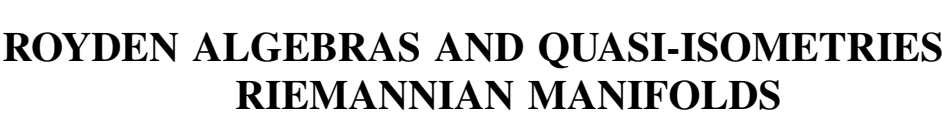




\title{
ROYDEN ALGEBRAS AND QUASI-ISOMETRIES OF RIEMANNIAN MANIFOLDS
}

\author{
Mitsuru NAKAI
}

The purpose of this paper is to investigate how the purely algebraic structure of the Royden algebra relates to the metric structure of the underlying Riemannian manifold. This investigation is motivated by the question: what potentialtheoretic properties are intrinsically determined by Royden algebras.

In order to develop the potential theory systematically on Riemannian 2-manifolds based on the Dirichlet principle, Royden [11, 12] introduced an algebra of continuous functions which now bears his name. A comprehensive survey of this algebra can be found in [14]. Royden algebras are equally powerful in the potential theory for higher dimensional Riemannian manifolds. (Cf. e.g. Nakai [6].)

In the 2-dimensional case we have seen (Nakai [4]) that the algebraic structure of the Royden algebra is characteristic of the quasiconformal structure of the manifold. In the present paper we shall show that in higher dimensional cases $(m \geqq 3)$ the algebraic structure of the Royden algebra is characteristic of the quasi-isometric structure of the underlying manifold. Our results will be precisely stated and a program for their proofs given in $\S 1$.

We will consider metric tensors, not necessarily continuous, by which Riemannian structures are given on manifolds. This seems to be the most appropriate frame work for classical potential theory, both from the view point of generality and for technical reasons.

\section{Main results.}

1. By a Riemannian manifold $M$ we mean a connected, separable, and orientable $m$-dimensional $(m \geqq 2)$ differentiable manifold of class $C^{1}$ with fundamental tensor

$$
G=\left(\begin{array}{ccc}
g_{11} & \cdots & g_{1 m} \\
\vdots & & \vdots \\
g_{m 1} & \cdots & g_{m m}
\end{array}\right)
$$

satisfying the following conditions:

In each parametric ball or cube ${ }^{1} B$ with local parameter $x=$

${ }^{1}$ If we use the terms parametric ball $(B, x)$, parametric cube $(B, x)$, or parametric region $(B, x)$, we always suppose that $\bar{B}$ is compact in $M$ and $x$ is defined in a neighborhood of $\bar{B}$. We also say that $B$ itself is a parametric ball, cube, or region if $x$ is clearly understood. 
$\left(x^{1}, \cdots, x^{m}\right)$, the local expressions $g_{i j}(x)$ of $g_{i j}(i, j=1, \cdots, m)$ of functions of $x$ are Borel measurable in $B$ and there exists a finite constant $k_{B} \geqq 1$ such that

$$
k_{B}^{-1} \sum_{i=1}^{m}\left(\xi^{i}\right)^{2} \leqq \sum_{i, j=1}^{m} g_{i j}(x) \xi^{i} \xi^{j} \leqq k_{B} \sum_{i=1}^{m}\left(\xi^{i}\right)^{2}
$$

for every $x$ in $B$ and for every $m$-tuple $\left(\xi^{1}, \cdots, \xi^{m}\right)$ of real numbers. Moreover there exists a covering $\{B\}$ of $M$ consisting of parametric balls or cubes $B$ such that

$$
1 \leqq k_{B} \leqq \tau
$$

for every $B$ of the covering and for some constant $\tau \in(1, \infty)^{2}$.

We can, therefore, consider the inverse matrix $G^{-1}$ of $G$. We set

$$
G^{-1}=\left(\begin{array}{ccc}
g^{11} & \cdots & g^{1 m} \\
\vdots & & \vdots \\
g^{m 1} & \cdots & g^{m m}
\end{array}\right), \quad g=\operatorname{det} G=\left|\begin{array}{ccc}
g_{11} & \cdots & g_{1 m} \\
\vdots & & \vdots \\
g_{m 1} & \cdots & g_{m m}
\end{array}\right| \text {. }
$$

In terms of the local parameter $x=\left(x^{1}, \cdots, x^{m}\right)$, the line element $d s$ on $M$ is given by $d s^{2}=\sum_{i, j=1}^{m} g_{i j}(x) d x^{i} d x^{j}$, and since the $g_{i j}(x)$ are Borel measurable, the line integral $\int_{\gamma} d s$ along any rectifiable curve $\gamma$ in $M$ can be defined. Therefore the natural distance $\rho_{M}(p, q)$ of two points $p$ and $q$ in $M$ is given by

$$
\rho_{M}(p, q)=\inf \int_{r} d s
$$

where the infimum is taken with respect to all rectifiable curves $\gamma$ in $M$ joining $p$ and $q$.

2. Let $M_{j}(j=1,2)$ be two Riemannian manifolds with the natural distances $\rho_{j}=\rho_{M_{j}}(j=1,2)$. A topological mapping $T$ of $M_{1}$ onto $M_{2}$ is said to be a quasi-isometry if there exists a finite constant $K \geqq 1$ such that

$$
K^{-1} \rho_{1}(p, q) \leqq \rho_{2}(T p, T q) \leqq K \rho_{1}(p, q)
$$

for any two points $p$ and $q$ in $M_{1}$. Clearly the inversemapping $T^{-1}$ of $T$ is also a quasi-isometry of $M_{2}$ onto $M_{1}$.

A quasi-isometry $T$ is necessarily a quasiconformal mapping, the latter being characterized by the existence of a finite constant $K \geqq 1$ such that

${ }^{2}$ If $\left(g_{i j}\right)$ is a continuous positive symmetric tensor, then (1) and (2) are automatically satisfied. Actually, in this case $\tau$ may be chosen as close to 1 as we wish. 


$$
\limsup _{r \rightarrow 0} \frac{\max _{\rho_{i}\left(p, p_{0}\right)=r} \rho_{j}\left(T_{i} p, T_{i} p_{0}\right)}{\min _{\rho_{i}\left(p, p_{0}\right)=r} \rho_{j}\left(T_{i} p, T_{i} p_{0}\right)} \leqq K
$$

at every point $p_{0}$ in $M_{i}$, where $(i, j)=(1,2)$ or $(2,1)$, and $T_{1}=T$, $T_{2}=T^{-1}$.

3. A function $f$ defined on a parametric cube $B$ : $a^{i}<x^{i}<b^{i}$ $(i=1, \cdots, m)$ is said to be absolutely continuous on lines (abbreviated as ACL) if it is absolutely continuous on almost all line segments parallel to coordinate axes; explicitly, if we denote by $B_{i}$ the face of $B$ given by $x^{i}=a^{i}$, then the function $f\left(\zeta+\xi e_{i}\right), e_{i}=\left(\delta^{i 1}, \cdots, \delta^{i m}\right)$, is absolutely continuous in $\xi \in\left(a^{i}, b^{i}\right)$ for almost every $\zeta \in B_{i}$ with respect to the $(m-1)$-dimensional Lebesgue measure $(i=1, \cdots, m)$. A function $f$ on a Riemannian manifold $M$ is said to be $A C L$ if $f \mid B$ is $A C L$ for every parametric cube in $M$. For such a function $f$ on $M$ the Dirichlet integral $D_{M}(f)$ over $M$ is defined:

$$
D_{M}(f)=\int \cdots \int_{M} \sum_{i, j=1}^{m} g^{i j}(x) \frac{\partial f}{\partial x^{i}}(x) \frac{\partial f}{\partial x^{j}}(x) \sqrt{g(x)} d x^{1} \cdots d x^{m} .
$$

It may or may not be finite.

We denote by $\boldsymbol{R}(M)$ the class of bounded continuous $A C L$ functions $f$ on $M$ which have finite Dirichlet integrals $D_{M}(f)<\infty$. With the scalar multiplication $(\alpha \cdot f)(p)=\alpha \cdot f(p)$, the addition $\left(f_{1}+f_{2}\right)=$ $f_{1}(p)+f_{2}(p)$, and the multiplication $(f \cdot g)(p)=f(p) \cdot g(p)$ as its algebraic operations, the class $\boldsymbol{R}(M)$ constitutes an algebra over the field of real numbers, called the Royden algebra associated with $M$. We are interested in the algebraic structure of $\boldsymbol{R}(M)$. Specifically, we ask how the algebraic structure of $\boldsymbol{R}(M)$ relates to the metric structure of $M$.

4. In a previous paper [4] we saw that if $m=2$, then the algebraic structure of the Royden algebra $\boldsymbol{R}(M)$ determines and is determined by the quasiconformal structure of $M$ :

Theorem. Let $M_{1}$ and $M_{2}$ be 2-dimensional Riemannian manifolds. If there exists a quasiconformal mapping $T$ of $M_{1}$ onto $M_{2}$, then the mapping $f \rightarrow f \circ T^{-1}$ is an algebraic isomorphism of $\boldsymbol{R}\left(M_{1}\right)$ onto $\boldsymbol{R}\left(M_{2}\right)$. Conversely, if there exists an algebraic isomorphism $\sigma$ : $f \rightarrow f^{\sigma}$ of $\boldsymbol{R}\left(M_{1}\right)$ onto $\boldsymbol{R}\left(M_{2}\right)$, then there exists a unique quasiconformal mapping $T$ of $M_{1}$ onto $M_{2}$ such that $f^{\sigma}=f \circ T^{-1}$ for every $f$ in $\boldsymbol{R}\left(M_{1}\right)$.

However, in [4], this theorem was proved for Riemann surfaces $M_{1}$ and $M_{2}$. It is not necessarily possible to give conformal structures to the $M_{i}$ compatible with the original metric structures of the $M_{i}$ 
in our present general setting. Therefore, to prove the above theorem, we need a minor modification of the discussion given in [4].

5. In view of the above, our main interest in the present paper is to study what happens when $m \geqq 3$. In contrast with the preceding theorem we shall show that if $m \geqq 3$, then the algebraic structure of the Royden algebra $\boldsymbol{R}(M)$ determines and is determined by the quasiisometric structure of $M$ :

THEOREM. Let $M_{1}$ and $M_{2}$ be Riemannian manifolds whose dimensions are greater than or equal to 3 . If there exists a quasiisometry $T$ of $M_{1}$ onto $M_{2}$, then the mapping $f \rightarrow f \circ T^{-1}$ is an algebraic isomorphism of $\boldsymbol{R}\left(M_{1}\right)$ onto $\boldsymbol{R}\left(M_{2}\right)$. Conversely, if there exists an algebraic isomorphism $\sigma: f \rightarrow f^{\sigma}$ of $\boldsymbol{R}\left(M_{1}\right)$ onto $\boldsymbol{R}\left(M_{2}\right)$, then there exists a unique quasi-isometry $T$ of $M_{1}$ onto $M_{2}$ such that $f^{\sigma}=f \circ T^{-1}$ for every $f$ in $\boldsymbol{R}\left(M_{1}\right)$.

In the above theorem, we do not make, for the existence of an algebraic isomorphism $\sigma$ of $\boldsymbol{R}\left(M_{1}\right)$ onto $\boldsymbol{R}\left(M_{2}\right)$, the priori assumption that the dimensions of $M_{1}$ and $M_{2}$ are the same; this will be a consequence.

6. For the proofs of Theorems in 4 and 5 , it is convenient and of independent interest to consider the following class of mappings: A Dirichlet mapping is a topological mapping $T$ of $M_{1}$ onto $M_{2}$, with the property that $f \in \boldsymbol{R}\left(M_{1}\right)$ if and only if $f_{0} T^{-1} \in \boldsymbol{R}\left(M_{2}\right)$.

We will see in $\S 2$ that for any Dirichlet mapping $T$ there exists a finite constant $K \geqq 1$ such that

$$
K^{-1} D_{M_{1}}(f) \leqq D_{M_{2}}\left(f \circ T^{-1}\right) \leqq K D_{M_{1}}(f)
$$

for every $f \in \boldsymbol{R}\left(M_{1}\right)$.

By means of inequality (7) we will prove in $\S 3$ that a mapping $T$ of $M_{1}$ onto $M_{2}$ is a Dirichlet mapping if and only if $T$ is a quasiconformal mapping $\left(\operatorname{dim} M_{1}=\operatorname{dim} M_{2}=2\right)$ or a quasi-isometry $\left(\operatorname{dim} M_{1}=\right.$ $\operatorname{dim} M_{2} \geqq 3$ ). This result in the 2-dimensional case has long: been known at least for Riemann surfaces if Dirichlet mappings are supposed to satisfy (7). Thus the emphasis here is again on the characterization of Dirichlet mappings as quasi-isometries in the higher dimensional case.

Dirichlet mappings can also be characterized as mappings which preserve Sobolev $(1,2)$-spaces on Riemannian manifolds with $m \geqq 3$. This will be discussed in $\S 4$.

Our main theorems in 4 and 5 above can be expressed in the 
following unified form: An algebraic isomorphism of $\boldsymbol{R}\left(M_{1}\right)$ onto $\boldsymbol{R}\left(M_{2}\right)$ induces and is induced by a Dirichlet mapping of $M_{1}$ onto $M_{2}$. This will be proved in $\S 5$.

\section{Royden algebras.}

7. In the Royden algebra $\boldsymbol{R}(M)$ associated with a Riemannian manifold $M$, there are the supremum norm $\|f\|_{\infty}=\sup _{p \in M}|f(p)|$ and the Dirichlet seminorm $\sqrt{D_{M}(f)}$. Combining these two norms we produce the third norm

$$
\|f\|=\|f\|_{\infty}+\sqrt{\overline{D_{M}(f)}} .
$$

We also write $\|f\|=\|f\|_{M}$ and $\|f\|_{\infty}=\|f\|_{\infty M}$ when an indication of the dependence on $M$ is needed. The norm (8) satisfies, in addition to the usual norm property, the relations $\|1\|=1$ and $\left\|f_{1} \cdot f_{2}\right\| \leqq$ $\left\|f_{1}\right\| \cdot\left\|f_{2}\right\|$ for all $f_{1}$ and $f_{2}$ in $\boldsymbol{R}(M)$. Therefore $(\boldsymbol{R}(M)$, $\|\cdot\|)$ constitutes a normed algebra. Moreover we have the following.

Proposition. $(\boldsymbol{R}(M),\|\cdot\|)$ is a Banach algebra.

What we have to show is the completeness of $\boldsymbol{R}(M)$ with respect to the norm $\|\cdot\|$. Let $\left\{f_{n}\right\}_{1}^{\infty}$ be a $\|\cdot\|$-Cauchy sequence in $\boldsymbol{R}(M)$. Since it is also $\|\cdot\|_{\infty}$-Cauchy, we can find a bounded continuous function $f$ on $M$ such that $\left\|f_{n}-f\right\|_{\infty} \rightarrow 0(n \rightarrow \infty)$.

Consider the Hilbert space $H(M)$ of Lebesgue measurable 1-forms $\alpha=\sum_{i=1}^{m} a^{i}(x) d x^{i}$ with

$$
\int \cdots \int_{M} \sum_{i, j=1}^{m} g^{i j}(x) a_{i}(x) a_{j}(x) \sqrt{g(x)} d x^{1} \cdots d x^{m}<\infty .
$$

The inner product $(\alpha, \beta)$ of $\alpha$ and $\beta=\sum_{i=1}^{m} b_{i}(x) d x^{i}$ is given by

$$
(\alpha, \beta)=\int \cdots \int_{M} \sum_{i, j=1}^{m} g^{i j}(x) a_{i}(x) b_{j}(x) \sqrt{g(x)} d x^{1} \cdots d x^{m} .
$$

Clearly $d f_{n}=\sum_{i=1}^{m}\left(\partial f_{n} / \partial x^{i}\right) d x^{i}$ belongs to $H(M)$ and $\left\{d f_{n}\right\}_{1}^{\infty}$ is a Cauchy sequence in $H(M)$, since

$$
\left(d f_{n}-d f_{n+p}, d f_{n}-d f_{n+p}\right)=D_{M}\left(f_{n}-f_{n-p}\right) \leqq\left\|f_{n}-f_{n+p}\right\| \cdot
$$

Let $\alpha=\sum_{i=1}^{m} a_{i}(x) d x^{i}$ be the limit of $\left\{d f_{n}\right\}$ in $H(M)$.

Take an arbitrary parametric cube $(B, x)$ and let $k_{B} \geqq 1$ be the constant in (1) for $B$. Then

$$
k_{B}^{-1} \sum_{i=1}^{m} \xi_{i}^{2} \leqq \sum_{i, j=1}^{m} g^{i j}(x) \xi^{i} \xi^{j} \leqq k_{B} \sum_{i=1}^{m} \xi_{i}^{2}, k_{B}^{-m} \leqq g(x) \leqq k_{B}^{m}
$$

for almost every $x \in B$ and for every $m$-tuple $\left(\xi_{1}, \cdots, \xi_{m}\right)$ of real numbers. In view of this 


$$
\int \cdots \int_{B} \sum_{i=1}^{m}\left(\frac{\partial f_{n}}{\partial x^{i}}(x)-a_{i}(x)\right)^{2} d x^{1} \cdots d x^{m} \leqq k_{B}^{m / 2+1}\left(d f_{n}-\alpha, d f_{n}-\alpha\right) .
$$

Therefore the left-hand member converges to zero as $n$ tends to infinity.

Let $B=\left\{x \mid c^{i}<x^{i}<d^{i}, i=1, \cdots, m\right\}$ and let $\pi_{i}$ be the largest subset of the face of $B$ determined by $x^{i}=c^{i}$ such that every line $L$ perpendicular to $x^{i}=c^{i}$ and passing through a point in $\pi_{i}$ possesses the following properties: every $f_{n}(n=1,2, \cdots)$ is absolutely continuous on $L \cap B$, and the $a_{i}$ are integrable on $L$. In view of the $A C L$ property of $f_{n}$, we can easily see, by the Fubini theorem, that the complement of $\pi_{i}$ in the face of $B$ containing $\pi_{i}$ is of $(m-1)$-dimensional Lebesgue measure zero. For each $x_{0 i}=\left(x_{0}^{1}, \cdots, x_{0}^{i-1}, x^{i}, x_{0}^{i+1}, \cdots\right.$, $\left.x_{0}^{m}\right) \in L \cap B$ and $x_{0}=\left(x_{0}^{1}, \cdots, x_{0}^{m}\right) \in L \cap \pi_{i}$, we obtain

$$
f_{n}\left(x_{0 i}\right)-f_{n}\left(x_{0}\right)=\int_{x_{0}^{i}}^{x^{i}} \frac{\partial f_{n}}{\partial x^{i}}\left(x_{0}^{1}, \cdots, x_{0}^{i-1}, t, x_{0}^{i+1}, \cdots, x_{0}^{m}\right) d t .
$$

Since $a_{i}$ is integrable on $L \cap B$

$$
\varphi\left(x_{0 i}\right)=\int_{x_{0}^{i}}^{x^{i}} a_{i}\left(x_{0}^{1}, \cdots, x_{0}^{i-1}, t, x_{0}^{i+1}, \cdots, x_{0}^{m}\right) d t
$$

can be defined. By the Schwarz inequality and the Fubini theorem, we obtain

$$
\begin{gathered}
\int \cdots \int_{\pi_{i}}\left|f_{n}\left(x_{0 i}\right)-f_{n}\left(x_{0}\right)-\varphi\left(x_{0 i}\right)\right|^{2} d x_{0}^{1} \cdots d x_{0}^{i-1} d x_{0}^{i+1} \cdots d x_{0}^{m} \\
\leqq\left(x^{i}\right)^{2} \int \cdots \int_{B}\left|\frac{\partial f_{n}}{\partial x^{i}}(x)-a_{i}(x)\right|^{2} d x^{1} \cdots d x^{m} .
\end{gathered}
$$

On letting $n \rightarrow \infty$, we conclude that

$$
\int \cdots \int_{\pi_{i}}\left|f_{n}\left(x_{0 i}\right)-f_{n}\left(x_{0}\right)-\varphi\left(x_{0 i}\right)\right|^{2} d x_{0}^{1} \cdots d x_{0}^{i-1} d x_{0}^{i+1} \cdots d x_{0}^{m}=0 .
$$

From this we obtain

$$
\begin{gathered}
f\left(x_{0}^{1}, \cdots, x_{0}^{i-1}, x^{i}, x_{0}^{i+1}, \cdots, x_{0}^{m}\right)-f\left(x_{0}^{1}, \cdots, x_{0}^{m}\right) \\
=\varphi\left(x_{0}^{1}, \cdots, x_{0}^{i-1}, x^{i}, x_{0}^{i+1}, \cdots, x_{0}^{m}\right)
\end{gathered}
$$

for every rational $x^{i}$ and for every $\left(x_{0}^{1}, \cdots, x_{0}^{i-1}, x_{0}^{i+1}, \cdots, x_{0}^{m}\right) \in \pi_{i}^{\prime} \subset \pi_{i}$ with $\pi_{i}-\pi_{i}^{\prime}$ of $(m-1)$-dimensional Lebesgue measure zero. Since both members of the above equality are continuous in $x^{i}$, we conclude that

$$
\begin{gathered}
f\left(x_{0}^{1}, \cdots, x_{0}^{i-1}, x^{i}, x_{0}^{i+1}, \cdots, x_{0}^{m}\right)-f\left(x_{0}^{1}, \cdots, x_{0}^{m}\right) \\
=\int_{x_{0}^{i}}^{x^{i}} a_{i}\left(x_{0}^{1}, \cdots, x_{0}^{i-1}, t, x_{0}^{i+1}, \cdots, x_{0}^{m}\right) d t
\end{gathered}
$$


for every $x^{i} \in\left(c^{i}, d^{i}\right)$ and $\left(x_{0}^{1}, \cdots, x_{0}^{i-1}, x_{0}^{i+1}, \cdots, x_{0}^{m}\right) \in \pi_{i}^{\prime}$. Since this is true for every $i=1, \cdots, m, f$ is $A C L$ on $B$ and $a_{i}=\partial f / \partial x^{i}(i=1, \cdots, m)$ almost everywhere on $B$. Therefore $f \in \boldsymbol{R}(M)$ and $\left\|f_{n}-f\right\| \rightarrow 0(n \rightarrow \infty)$.

8. We denote by $C^{1}(M)$ the class of $C^{1}$-functions on $M$. The following will be useful in calculations:

Proposition. $\boldsymbol{R}(M) \cap C^{1}(M)$ is dense in $\boldsymbol{R}(M)$ in the norm $\|\cdot\|$.

For an arbitrary $f \in \boldsymbol{R}(M)$ and a positive number $\varepsilon$, we have to find a $\psi$ in $R(M) \cap C^{1}(M)$ with $\|f-\psi\|<\varepsilon$. Let $\left\{\varphi_{n}\right\}_{1}^{\infty} \subset C^{1}(M)$ be a partition of unity with the following properties: the support of each $\varphi_{n}$ is contained in a parametric ball $\left(B_{n}, x\right)$; for each compact set $K$ in $M, K \cap B_{n}=\varnothing$ except for a finite number of $n ; \sum_{1}^{\infty} \varphi_{n}=1$ on $M$. Observe that $\varphi_{n} f \in \boldsymbol{R}(M)(n=1,2, \cdots)$. If we can find a $\psi_{n} \in C^{1}(M)$ with its support in $B_{n}$ such that $\left\|\varphi_{n} f-\psi_{n}\right\|<\varepsilon / 2^{n}$, then $\psi=\sum_{1}^{\infty} \psi_{n}$ is well-defined and belongs to $C^{1}(M)$. Since

$$
\|f-\psi\|=\left\|\sum_{n=1}^{\infty}\left(\varphi_{n} f-\psi_{n}\right)\right\| \leqq \sum_{n=1}^{\infty}\left\|\varphi_{n} f-\psi_{n}\right\|<\varepsilon,
$$

we see that $\psi \in C^{1}(M) \cap \boldsymbol{R}(M)$, i.e. $\psi$ is the required function.

In view of the above, we have only to consider an $f$ in $R(M)$ whose support is contained in a parametric ball $(B, x)$. By the regularization method (cf. e.g. Yoshida $[17 ;$ p. 29,58$]$ ), we can find a $C^{1}$ function $\psi$ in $B$ with its support in $B$ such that

$$
\begin{aligned}
\|f-\psi\|_{\infty, B}+ & \left(\int \cdots \int_{B} \sum_{i=1}^{m}\left|\frac{\partial f}{\partial x^{i}}(x)-\frac{\partial \psi}{\partial x^{i}}(x)\right|^{2} d x^{1} \cdots d x^{m}\right)^{1 / 2} \\
& <\varepsilon k_{B}^{-m / 2-1}
\end{aligned}
$$

where $k_{B}$ is the constant in (1) for $B$. From this we conclude that $\|f-\psi\|=\|f-\psi\|_{B}<\varepsilon$.

9. For two functions $f_{1}$ and $f_{2}$ on $M$, we define the lattice operations $U$ and $\cap$ by $\left(f_{1} \cup f_{2}\right)(p)=\max \left(f_{1}(p), f_{2}(p)\right)$ and $\left(f_{1} \cap f_{2}\right)(p)=$ $\min \left(f_{1}(p), f_{2}(p)\right)$. The following property of $\boldsymbol{R}(M)$ is important for our purposes:

Proposition. $\boldsymbol{R}(M)$ is closed under the lattice operations $U$ and $\cap$, and

$$
\begin{gathered}
D_{M}(f)=D_{M}(f \cap c)+D_{M}(f \cup c), \\
D_{(f=c)}(f)=D_{(f=c)}(f \cap c)=D_{(f=c)}(f \cup c)=0
\end{gathered}
$$

for every functions $f$ in $\boldsymbol{R}(M)$ and every real number $c$. 
Observe that $f_{1} \cup f_{2}=\left(f_{1}-f_{2}\right) \cup 0+f_{2}$ and $f_{1} \cap f_{2}=-\left(\left(-f_{1}\right) \cup\left(-f_{2}\right)\right)$. Therefore to prove that $\boldsymbol{R}(M)$ is closed under the lattice operations, it is sufficient to show that $f \cup 0 \in \boldsymbol{R}(M)$ for every $f \in \boldsymbol{R}(M)$. If $f$ is absolutely continuous on a line segment in a parametric ball, then $f \cup 0$ has the same property. Therefore $f \cup 0$ is $A C L$ on $M$ along with $f$. It is also clear that if both $\partial f / \partial x^{i}$ and $\partial(f \cup 0) / \partial x^{i}$ exist at $x$, then

$$
\left|\frac{\partial(f \cup 0)}{\partial x^{i}}(x)\right| \leqq\left|\frac{\partial f}{\partial x^{i}}(x)\right| \text {. }
$$

Therefore $D_{M}(f \cup 0) \leqq D_{M}(f)$ and we conclude that $f \cup 0 \in \boldsymbol{R}(M)$.

It is easy to see that (11) implies (10). To prove (11) we only have to treat the case $c=0$. In view of (12), both $D_{(f=0)}(f \cup 0)$ and $D_{(f=0)}(f \cap 0)$ are dominated by $D_{(f=0)}(f)$. Hence we have to prove that $D_{(f=0)}(f)=0$. Let $(B, x)$ be an arbitrary parametric cube. In view of

$$
D_{(f=0) \cap B}(f) \leqq k_{B}^{m / 2+1} \int \cdots \int_{(f=0) \cap B} \sum_{i=1}^{m}\left|\frac{\partial f}{\partial x^{i}}(x)\right|^{2} d x^{1} \cdots d x^{m},
$$

it is sufficient to show that

$$
\int \cdots \int_{(f=0) \cap B}\left|\frac{\partial f}{\partial x^{i}}(x)\right|^{2} d x^{2} \cdots d x^{m}=0
$$

for every $i=1, \cdots, m$. Let $B=\left\{x \mid c^{i}<x^{i}<d^{i}, i=1, \cdots, m\right\}$ and let $\pi_{i}$ be the largest subset of the face of $B$ determined by $x^{i}=c^{i}$ such that, for every line $L$ perpendicular to $x^{i}=c^{i}$ and passing through a point in $\pi_{i}, f(x)$ is absolutely continuous on $L \cap B$. Since the complement of $\pi_{i}$ with respect to the face of $B$ containing $\pi_{i}$ is of $(m-1)$ Lebesgue measure zero, the Fubini theorem assures that (13) is equivalent to

$$
\int \cdots \int_{\pi_{i}}\left(\int_{(f=0) \cap\left[c^{i}, d^{i}\right]}\left|\frac{\partial f}{\partial x^{i}}(x)\right|^{2} d x^{i}\right) d x^{1} \cdots d x^{i-1} d x^{i+1} \cdots d x^{m}=0 .
$$

Thus to prove (12) it is sufficient to show that

$$
\int_{E} \varphi^{\prime}(t)^{2} d t=0
$$

where $\varphi(t)$ is absolutely continuous in an interval $[c, d]$ and $E=$ $\{t \in[a, b] \mid \varphi(t)=0\}$. Let $E_{1}$ be the largest subset of $E$ such that $\varphi^{\prime}(t)$ exists for each $t \in E_{1}$. Put $E_{2}=\left\{t \in E_{1} \mid \varphi^{\prime}(t)=0\right\}$. Pick an arbitrary point $t_{0}$ in $E_{1}-E_{2}$. Since $\varphi\left(t_{0}\right)=0$ and $\varphi^{\prime}\left(t_{0}\right) \neq 0, t_{0}$ is an isolated point in $E$. This shows that $E_{1}-E_{2}$ is at most countably infinite and a fortiori $E_{1}-E_{2}$ has Lebesgue measure zero. Since $E-E_{1}$ also has Lebesgue measure zero, $\varphi^{\prime}(t)=0$ almost everywhere on $E$. We conclude that (14) is valid. 
10. Recall that a homeomorphism $T$ of a Riemannian manifold $M_{1}$ onto another $M_{2}$ is a Dirichlet mapping if $f \in \boldsymbol{R}\left(M_{1}\right)$ is equivalent to $f \circ T^{-1} \in R\left(M_{2}\right)$. We prove here that the induced mapping $f \rightarrow f \circ T^{-1}$ of $\boldsymbol{R}\left(M_{1}\right)$ onto $\boldsymbol{R}\left(M_{2}\right)$ has the following continuity property (cf. Nakai [5]):

Theorem. For any Dirichlet mapping $T$ of a Riemannian manifold $M_{1}$ onto another $M_{2}$, there exists a finite constant $K \geqq 1$ such that

$$
K^{-1} D_{M_{1}}(f) \leqq D_{M_{2}}\left(f \circ T^{-1}\right) \leqq K D_{M_{1}}(f)
$$

for every function $f$ in $\boldsymbol{R}\left(M_{1}\right)$.

For simplicity let $S=T^{-1}$ and $S^{*} f=f \circ S$. Clearly $S^{*}$ is an algebraic isomorphism of $\boldsymbol{R}\left(M_{1}\right)$ onto $\boldsymbol{R}\left(M_{2}\right)$. Since $\boldsymbol{R}\left(M_{i}\right)$ are Banach algebras $(i=1,2), S^{*}$ must be bicontinuous in the norm $\|\cdot\|$ (cf. e.g. Rickart [9]). Hence there exists a finite constant $K \geqq 1$ such that

$$
K^{-1}\|f\|_{M_{1}} \leqq\|f\|_{M_{2}} \leqq K\|f\|_{M_{1}}
$$

for every function $f$ in $\boldsymbol{R}\left(M_{1}\right)$. Observe that

$$
\left\|S^{*} f\right\|_{\infty, M_{2}}=\|f\|_{\infty, M_{1}}, \quad S^{*}\left(f_{1} \cup f_{2}\right)=\left(S^{*} f_{1}\right) \cup_{\cap}\left(S^{*} f_{2}\right)
$$

for every $f, f_{1}$, and $f_{2}$ in $\boldsymbol{R}\left(M_{1}\right)$.

Let $F=\left\{f \in R\left(M_{1}\right) \mid 0 \leqq f \leqq 1\right\}$. From (16) it follows that

$$
\sqrt{D_{M_{2}}\left(S^{*} f\right)} \leqq K\left(1+\sqrt{D_{M_{1}}(f)}\right)
$$

for every $f \in F$. For an arbitrarily fixed function $f$ in $F$, the functions

$$
f_{i}=n\left(\left(\frac{i-1}{n} \cup f\right) \cap \frac{i}{n}-\frac{i-1}{n}\right) \quad(i=1, \cdots, n)
$$

are all in $F$. In view of (17),

$$
S^{*} f_{i}=n\left(\left(\frac{i-1}{n} \cup\left(S^{*} f\right)\right) \cap \frac{i}{n}-\frac{i-1}{n}\right) \quad(i=1, \cdots, n) .
$$

Here we have used the relation $S^{*} c=c$ for every constant $c$. Observe that

$$
\begin{aligned}
D_{M_{1}}\left(f_{i}\right) & =n^{2} D_{M_{1}}\left(\left(\frac{i-1}{n} \cup f\right) \cap \frac{i}{n}\right), \\
D_{M_{2}}\left(S^{*} f_{i}\right) & =n^{2} D_{M_{2}}\left(\left(\frac{i-1}{n} \cup\left(S^{*} f\right)\right) \cap \frac{i}{n}\right)
\end{aligned}
$$

for every $i=1, \cdots, n$. Repeated use of (9) yields 


$$
D_{M_{1}}(f)=\sum_{i=1}^{n} D_{M_{1}}\left(\left(\frac{i-1}{n} \cup f\right) \cap \frac{i}{n}\right)
$$

and

Therefore

$$
D_{M_{2}}\left(S^{*} f\right)=\sum_{i=1}^{n} D_{M_{2}}\left(\left(\frac{i-1}{n} \cup\left(S^{*} f\right)\right) \cap \frac{i}{n}\right) .
$$

$$
n^{2} D_{M_{1}}(f)=\sum_{i=1}^{n} D_{M_{1}}\left(f_{i}\right), \quad n^{2} D_{M_{2}}\left(S^{*} f\right)=\sum_{i=1}^{n} D_{M_{2}}\left(S^{*} f_{i}\right) .
$$

Since $f_{i} \in F(i=1, \cdots, m),(18)$ implies

$$
D_{M_{2}}\left(S^{*} f_{i}\right) \leqq K^{2}\left(1+2 \sqrt{\overline{D_{M_{1}}\left(f_{i}\right)}}+D_{M_{1}}\left(f_{i}\right)\right) \quad(i=1, \cdots, n) .
$$

On summing these inequalities with respect to $i=1, \cdots, n$, we obtain by (19)

$$
n^{2} D_{M_{2}}\left(S^{*} f\right) \leqq K^{2}\left(n+2 \sum_{i=1}^{n} \sqrt{D_{M_{1}}\left(f_{i}\right)}+n^{2} D_{M_{1}}(f)\right) .
$$

Let $i(n)$ be a fixed integer such that $1 \leqq i(n) \leqq n$ and

We set

$$
D_{M_{1}}\left(f_{i(n)}\right)=\max _{1 \leqq i \leqq n} D_{M_{1}}\left(f_{i}\right) \text {. }
$$

$$
u_{n}=\left(\frac{i(n)-1}{n} \cup f\right) \cap \frac{i(n)}{n} .
$$

The above inequality takes on the following form:

$$
D_{M_{2}}\left(S^{*} f\right) \leqq K^{2} D_{M_{1}}(f)+n^{-1} K^{2}+2 K^{2} \sqrt{D_{M_{1}}\left(u_{n}\right)} .
$$

Choose a subsequence $\{n(k)\}_{k=1}^{\infty}$ of the sequence of positive integers such that $a=\lim _{k \rightarrow \infty} i(n(k)) / n(k)$ exists. Fix an arbitrary positive integer $N$. Since

$$
a-\frac{1}{N}<\frac{i(n(k))-1}{n(k)}<\frac{i(n(k))}{n(k)}<a+\frac{1}{N}
$$

for all sufficiently large $k$, we obtain by (10)

$$
\operatorname{limsum}_{k \rightarrow \infty} D_{M_{1}}\left(u_{n(k)}\right) \leqq D_{(a-1 / N<f<a+1 / N)}(f) .
$$

In view of (11), we conclude that

$$
\lim _{N \rightarrow \infty} D_{(a-1 / N<f<a+1 / N)}(f)=D_{(f=a)}(f)=0
$$

and therefore

$$
\lim _{k \rightarrow \infty} D_{M_{1}}\left(u_{n(k)}\right)=0 .
$$

On letting $k \rightarrow \infty$ in (20) with $n=n(k)$, we infer that (21) implies (15). 


\section{Dirichlet mappings.}

11. In this section we will prove the following geometric characterization of Dirichlet mappings:

THEOREM. A homeomorphism $T$ of a Riemannian manifold $M_{1}$ onto another $M_{2}$ is a Dirichlet mapping if and only if $T$ is a quasiconformal mapping of $M_{1}$ onto $M_{2}$ for $\operatorname{dim} M_{1}=\operatorname{dim} M_{2}=2$ and $a$ quasi-isometry of $M_{1}$ onto $M_{2}$ for $\operatorname{dim} M_{1}=\operatorname{dim} M_{2} \geqq 3$.

The proof will be given in 12-16. The theorem in the 2-dimensional case is well-known for plane regions $M_{i}$. In the higher dimensional case it was proved in Nakai [7] for regions $M_{i}$ in the $m$-dimensional Euclidean space $(m \geqq 3)$. We will show how to modify the proofs for the present situation.

12. In view of (2), we can find a countable covering $\left\{B_{i}\right\}_{1}^{\infty}$ of $M_{1}$ consisting of parametric regions $B_{i}$ homeomorphic to a sphere with the following properties: $T B_{i}(i=1,2, \cdots)$ are parametric regions on $M_{2}$ and

$$
\max \left(k_{B_{i}}, k_{T_{i}}\right) \leqq \tau \quad(i=1,2, \cdots)
$$

where $\tau$ is a constant in $(1, \infty)$ and $k_{B_{i}}$ and $k_{T B_{i}}$ are the constants in (1) for $B_{i}$ and $T B_{i}$. We denote by $D_{B}(\varphi)$ the Euclidean Dirichlet integral of a function $\phi$ on a parametric region $B$, i.e.

$$
\boldsymbol{D}_{B}(\varphi)=\int \cdots \int_{B} \sum_{i=1}^{m}\left|\frac{\partial \varphi}{\partial x^{i}}(x)\right|^{2} d x^{1} \cdots d x^{m} .
$$

13. In nos. 13 and 14, we assume that $\operatorname{dim} M_{1}=\operatorname{dim} M_{2}=m=2$. First we assume that $T$ is a quasiconformal mapping of $M_{1}$ onto $M_{2}$, i.e. $T$ satisfies (5) for some $K \geqq 1$. Choose an arbitrary $B_{i}=B$ in 12. Then by (22)

$$
\limsup _{r \rightarrow 0} \frac{\max _{|h|=r}|T(x+h)-T(x)|}{\min _{|h|=r} \mid T(x+h)-T(x)} \leqq \tau^{2} K
$$

for every $x \in B$, with $|x|=\left|\left(x^{1}, \cdots, x^{m}\right)\right|=\sum_{i=1}^{m}\left|x^{i}\right|^{2}$. Therefore $T$ is a $\tau^{2} K$-quasiconformal mapping of $B$ onto $T B$ with respect to the Euclidean metric.

It is well-known (see e.g. Künzi [2], Gehring [1]) that (23) implies that $\varphi \circ T^{-1}$ is $A C L$ on $T B$ along with $\varphi$ on $B$ and

$$
\tau^{-2} K^{-1} \boldsymbol{D}_{B}(\varphi) \leqq \boldsymbol{D}_{T B}\left(\varphi \circ T^{-1}\right) \leqq \tau^{2} K \boldsymbol{D}_{B}(\varphi) \text {. }
$$

Again by (22), (24) gives 


$$
\tau^{-m-4} K^{-1} D_{B}(\varphi) \leqq D_{T B}\left(\varphi \circ T^{-1}\right) \leqq \tau^{m+4} K D_{B}(\varphi)
$$

where we have used the inequalities $k_{B}^{-1} \leqq\left(g_{i j}\right) \leqq k_{B}$ and $k_{B}^{-m} \leqq g \leqq k_{B}^{m}$. Since (25) is valid for every $B=B_{i}$, we conclude that $f \in \boldsymbol{R}\left(M_{1}\right)$ is equivalent to $f \circ T^{-1} \in \boldsymbol{R}\left(M_{2}\right)$.

14. Conversely assume that $T$ is a Dirichlet mapping of $M_{1}$ onto $M_{2}$. We have seen that (15) is valid for some $K$. Again fix an arbitrary $B=B_{i}$. Then (15) implies (24) for $\varphi \in \boldsymbol{R}\left(M_{1}\right)$ with compact support in $B$. In particular, we deduce that

$$
\tau^{-2} K \bmod A \leqq \bmod T A \leqq \tau^{2} K \bmod A
$$

for every ring (annulus) $A$ contained in $B$. Here $\bmod A$ means the harmonic modulus of $A$ given by

$$
\bmod A=\frac{2 \pi}{D_{A}\left(w_{A}\right)},
$$

where $w_{A}$ is the harmonic function on $A$ with boundary values 0 at the exterior boundary of $A$ and 1 at the interior boundary of $A$.

It is again well-known (see e.g. Künzi [2], Gehring [1]) that a homeomorphism $T$ of $B$ onto $T B$ with the property (26) is a $\tau^{2} K$ quasiconformal mapping of $B$ onto $T B$, i.e. (23) is valid. Since $B=B_{i}$ is arbitrary, we conclude that (5) holds with $K$ replaced by $\tau^{4} K$. Thus the theorem for $\operatorname{dim} M_{1}=\operatorname{dim} M_{2}=2$ is proved.

15. In nos. 15 and 16 , we assume that $\operatorname{dim} M_{1}=\operatorname{dim} M_{2}=m \geqq 3$. First suppose $T$ is a quasi-isometry of $M_{1}$ onto $M_{2}$, i.e. (4) is valid for some $K$. Fix a $B=B_{i}$. By (22), we obtain

$$
\tau^{-1} K^{-1}\left|x_{1}-x_{2}\right| \leqq\left|T x_{1}-T x_{2}\right| \leqq \tau K\left|x_{1}-x_{2}\right|
$$

for every $x_{1}$ and $x_{2}$ in $B$. It is clear that $\varphi \circ T^{-1}$ is $A C L$ on $T B$ along with $\varphi$ on $B$. By the classical Rademacher [10]-Stepanoff [15] theorem (see also Tsuji [16], Saks [13]), we can compute $D_{T B}\left(\varphi \circ T^{-1}\right)$ as follows:

$$
\boldsymbol{D}_{T B}\left(\varphi \circ T^{-1}\right)=\int \cdots \int_{B} \sum_{i=1}^{m}\left|\frac{\partial\left(\varphi \circ T^{-1}\right)}{\partial y^{i}}(y(x))\right|^{2}\left|J_{y}(x)\right| d x^{1} \cdots d x^{m},
$$

where $y=y(x)=T(x)$ and $J_{y}(x)=\operatorname{det}\left(\partial y^{j} / \partial x^{i}\right)$. Observe that

$$
\begin{aligned}
\left|\frac{\partial\left(\varphi \circ T^{-1}\right)}{\partial y^{i}}(y(x))\right|^{2} & =\left|\sum_{i=1}^{m} \frac{\partial \varphi}{\partial x^{j}}(x) \cdot \frac{\partial x^{j}}{\partial y^{i}}\right|^{2} \\
& \leqq \sum_{j=1}^{m}\left|\frac{\partial \varphi}{\partial x^{i}}(x)\right|^{2} \cdot \sum_{j=1}^{m}\left|\frac{\partial x^{j}}{\partial y^{i}}\right|^{2}
\end{aligned}
$$


almost everywhere (cf. Nakai [7]). In view of (27), we see that

$$
m^{-2} \tau^{-2} K^{-2} \leqq \sum_{i, j=1}^{m}\left|\frac{\partial x^{j}}{\partial y^{i}}\right|^{2} \leqq m^{2} \tau^{2} K^{2}
$$

almost everywhere and also

$$
(\tau K)^{-m} \leqq\left|J_{y}(x)\right| \leqq(\tau K)^{m}
$$

almost everywhere. Here we have repeatedly used the measurability of $T$ and $T^{-1}$, which is a consequence of the Rademacher-Stepanoff theorem cited above. From (28)-(31), it follows that

$$
m^{-2}(\tau K)^{-m-2} \boldsymbol{D}_{B}(\varphi) \leqq \boldsymbol{D}_{T B}\left(\varphi \circ T^{-1}\right) \leqq m^{2}(\tau K)^{m+2} \boldsymbol{D}_{B}(\varphi)
$$

for every $B=B_{i}$. Therefore (15) is valid with $K$ replaced by $m^{2}(\tau K)^{m+2}$. We conclude that $f \in \boldsymbol{R}\left(M_{1}\right)$ is equivalent to $f \circ T^{-1} \in \boldsymbol{R}\left(M_{2}\right)$.

16. We come to the final part of our proof. Assume that $T$ is a Dirichlet mapping of $M_{1}$ onto $M_{2}$. Since (15) is valid for some $K$, we see by (22) that

$$
K_{1}^{-1} \boldsymbol{D}_{B}(\varphi) \leqq \boldsymbol{D}_{T B}\left(\varphi \circ T^{-1}\right) \leqq K_{1} \boldsymbol{D}_{B}(\varphi)
$$

for every $B=B_{i}$ in 12 and every $\varphi \in R\left(M_{1}\right)$ with compact support in $B$, where $K_{1}=\tau K$. The proof in Nakai [7] can be applied to deduce from (32) that

$$
K_{2}^{-1}\left|x_{1}-x_{2}\right| \leqq\left|T x_{1}-T x_{2}\right| \leqq K_{2}\left|x_{1}-x_{2}\right|
$$

for every $x_{1}$ and $x_{2}$ in $B$ with $\left|x_{1}-x_{2}\right|$ sufficiently small. Here $K_{2}$ depends only on $K_{1}$ but not on $B$. Again by (22), we obtain (4) with $K=\tau K_{2}$ for every $p$ and $q$ in $M_{1}$ such that $\rho_{1}(p, q)$ is sufficiently small. By the definition (3) of $\rho_{1}$ and $\rho_{2}$, the requirement on the smallness of $\rho_{1}(p, q)$ can obviously be removed.

4. Sobolev $(1,2)$-spaces.

17. Let $W^{1,2}(M)$ be the class of functions $f$ on a Riemannian manifold $M$ such that the $\partial f / \partial x^{i}(i=1, \cdots, m=\operatorname{dim} M)$ exist almost everywhere on $M$ and

$$
\begin{aligned}
\|\| f \|_{M}= & \int \cdots \int_{M}\left(|f(x)|^{2}+\sum_{i, j=1}^{m} g^{i j}(x) \frac{\partial f}{\partial x^{i}}(x) \frac{\partial f}{\partial x^{j}}(x)\right) \\
& \cdot \sqrt{g(x)} d x^{1} \cdots d x^{m}<\infty .
\end{aligned}
$$

The class $W^{1,2}(M)$ is called the Sobolev $(1,2)$-space (cf. e.g. Yosida [17]). For simplicity, we write $W(M)=W^{1,2}(M)$.

Let $T$ be a homeomorphism of a Riemannian manifold $M_{1}$ onto 
another $M_{2}$. For a function $\phi$ on $M_{1}$, we define the function $T^{*} \phi$ on $M_{2}$ by

$$
T^{*} \varphi=\varphi \circ T^{-1} .
$$

THEOREM. For a homeomorphism $T$ of $M_{1}$ onto $M_{2}$ with $\operatorname{dim} M_{1}=$ $\operatorname{dim} M_{2} \geqq 3$, the following conditions are equivalent:

(a) $T$ is a Dirichlet mapping of $M_{1}$ onto $M_{2}$;

(b) $T$ is a quasi-isometry of $M_{1}$ onto $M_{2}$;

(c) $T^{*}\left(W\left(M_{1}\right)\right)=W\left(M_{2}\right)$.

The equivalence of (a) and (b) was established in $\S 3$. We only have to show that (c) is equivalent to (a) or (b). The proof is given in 18-19.

18. By the regularization method (cf. 8), we see that $W(M) \cap C^{1}(M)$ is dense in $W(M)$ with respect to the norm $\|\cdot\| \|$ and also $W(M) \cap C^{1}(M)$ is dense in $W(M) \cap C(M)$ with respect to the norm $\|\cdot\|$. From the latter assertion, we deduce

$$
\boldsymbol{R}(M)=W(M) \cap C(M) \cap L^{\infty}(M) .
$$

Here as usual $C$ stands for continuous and $L^{\infty}(M)$ is the set of essentially bounded functions on $M$. Of course $C(M) \cap L^{\infty}(M)$ is nothing but the class of bounded continuous functions on $M$. Therefore for every homeomorphism $T$ of $M_{1}$ onto $M_{2}$

$$
T^{*}\left(C\left(M_{1}\right) \cap L^{\infty}\left(M_{1}\right)\right)=C\left(M_{2}\right) \cap L^{\infty}\left(M_{2}\right) .
$$

From (36) and (37), we see that (c) implies $T^{*}\left(\boldsymbol{R}\left(M_{1}\right)\right)=\boldsymbol{R}\left(M_{2}\right)$, i.e. $T$ is a Dirichlet mapping.

19. Take an arbitrary $B=B_{i}$ in 12 . Let $y=y(x)$ be the representation of $T$ considered as a mapping of $B$ onto $T B$. We saw in 15 that there exists a constant $K$ independent of $B$ such that $K^{-1} \leqq J_{y}(x) \leqq K$ almost everwhere on $B$. Using the ||$\cdot|| \mid$-denseness of $W\left(M_{1}\right) \cap C^{1}\left(M_{1}\right)$ in $W\left(M_{1}\right)$, we can deduce a relation similar to (29) for an arbitrary $f \in W\left(M_{1}\right)$. We could have chosen the above constant $K$ so large that $K^{-1} \leqq \sum_{i, j=1}^{m}\left|\partial x^{j} / \partial y^{i}\right|^{2} \leqq K$. Then

$$
K^{-2} \boldsymbol{D}_{B}(f) \leqq \boldsymbol{D}_{T B}\left(T^{*} f\right) \leqq K^{2} \boldsymbol{D}_{B}(f)
$$

and also

$$
\begin{aligned}
K \int \cdots \int_{B}|f(x)|^{2} d x^{1} \cdots d x^{m} & \leqq \int \cdots \int_{B}\left|\left(T^{*} f\right)(y)\right|^{2} d y^{1} \cdots d y^{m} \\
& \leqq K \int \cdots \int_{B}|f(x)|^{2} d x^{1} \cdots d x^{m}
\end{aligned}
$$


By (22), we obtain

$$
\tau^{-m / 2-1} K^{-1}|||f|\left\|_{B} \leqq\right\||| T^{*} f \mid\left\|_{T_{B}} \leqq \tau^{m / 2+1} K\right\| f f \|_{B} .
$$

From this we see that $T^{*} W\left(M_{1}\right)=W\left(M_{2}\right)$.

\section{Royden compactifications.}

20. Let $p^{*}$ be an algebraic homomorphism of $\boldsymbol{R}(M)$ onto the field of real numbers such that $p^{*}(1)=1$. Denote by $M^{*}$ the set of all such $p^{*}$. We denote by $\boldsymbol{R}(M)^{*}$ the dual Banach space of $\boldsymbol{R}(M)$ endowed with the weak star topology. Take an arbitrary $f \in \boldsymbol{R}(M)$ and an arbitrary number $\lambda>\|f\|_{\infty}$. Observe that $\sqrt{\lambda \pm f} \in \boldsymbol{R}(M)$. For every $p^{*} \in M^{*}$, we have

$$
\lambda \pm p^{*}(f)=p^{*}(\lambda \pm f)=p^{*}\left((\sqrt{\lambda \pm f})^{2}\right)=\left(p^{*}(\sqrt{\lambda \pm f})^{2}\right) \geqq 0 .
$$

Hence $\left|p^{*}(f)\right| \leqq \lambda$ and a fortiori $\left|p^{*}(f)\right| \leqq\|f\|_{\infty} \leqq\|f\|$. Therefore $M^{*}$ is a bounded weakly star closed subspace of $\boldsymbol{R}(M)^{*}$. This shows that $M^{*}$ is weakly star compact. Each point $p \in M$ can be viewed as a point in $M^{*}$ by considering $p(f)=f(p)$ for $f \in \boldsymbol{R}(M)$. It is clear that the original topology of $M$ is identical with the relative weak star topology in $M \subset M^{*}$.

For $f \in \boldsymbol{R}(M)$, set $f\left(p^{*}\right)=p^{*}(f)$. Then $f \in C(M)$, the space of bounded continuous functions. We can thus consider $\boldsymbol{R}(M) \subset C\left(M^{*}\right)$. Since the Stone-Weierstrass property is satisfied by $\boldsymbol{R}(M)$, we conclude that $\boldsymbol{R}(M)$ is dense in $C\left(M^{*}\right)$ with respect to the supremum norm. Therefore $M$ is a dense subspace of $M^{*}$. Since $M$ is locally compact, $M^{*}-M$ cannot accumulate at any point in $M$, i.e. $M$ is open in $M^{*}$. We summarize:

The compact Hausdorff space $M^{*}$ contains $M$ as an open dense subspace; each function in $\boldsymbol{R}(M)$ can be uniquely extended to $M^{*}$ so as to be in $C\left(M^{*}\right) ; \boldsymbol{R}(M)$ separates points in $M^{*}$, i.e. for every $p_{1}^{*}$ and $p_{2}^{*}$ in $M^{*}$ with $p_{1}^{*} \neq p_{2}^{*}$, there exists an $f \in \boldsymbol{R}(M)$ such that $f\left(p_{1}^{*}\right) \neq f\left(p_{2}^{*}\right)$. Any compact Hausdorff space with these properties is identical with $M^{*}$.

We call $M^{*}$ the Royden compactification of $M$.

21. The Royden compactification is a convenient tool in the study of global potential theory on manifolds based on the Dirichlet principle. It is especially powerful in the classification problem (see Nakai [6]). However, here we are only interested in the topological structure of $M^{*}$. First we show (cf. Nakai [3]):

Proposition. A point $p^{*}$ in $M^{*}$ is in $M$ if and only if $\left\{p^{*}\right\}$ is 
$a G_{\dot{\delta}}$-set, i.e. the first countability axiom is satisfied at $p^{*}$.

If $p^{*} \in M$, then clearly $p^{*}$ is a $G_{\dot{\delta}}$-set. Suppose $p^{*}$ is a $G_{\dot{\delta}}$-set. Contrary to the assertion, assume $p^{*} \in M^{*}-M$. Then there would exist a sequence $\left\{p_{n}\right\}_{1}^{\infty} \subset M$ converging to $p^{*}$. Take parametric balls $\left(B_{n}, x_{n}\right) \quad(n=1,2, \cdots)$ with the following properties: $p_{n} \in B_{n}$ and $x\left(p_{n}\right)=0 ; \bar{B}_{n} \cap \bar{B}_{n^{\prime}}=\varnothing\left(n \neq n^{\prime}\right)$;

in each $B_{n}$

$$
\overline{\left(\bigcup_{n=1}^{\infty} \bar{B}_{n}\right)}-\bigcup_{n=1}^{\infty} \bar{B}_{n}=\left\{p^{*}\right\} \text {; }
$$

$$
\tau^{-1}\left(\delta_{i j}\right) \leqq\left(g_{i j}\right) \leqq \tau\left(\delta_{i j}\right)
$$

almost everywhere. Let $\left\{a_{n}\right\}_{1}^{\infty}$ be a sequence such that $0<a_{n}<2^{-n}$ and

$$
A_{n}= \begin{cases}\left\{x_{n}\left|a_{n}^{1 /(m-2)}<\right| x_{n} \mid<\left(2 a_{n}\right)^{1 / m-2}\right\} & (m \geqq 3) \\ \left\{x_{n}\left|e^{-1 / a_{n}} a_{n}<\right| x_{n} \mid<a_{n}\right\} & (m=2)\end{cases}
$$

is contained in $B_{n}$. Denote by $A_{n}^{\prime}$ the closed ball bounded by the inner boundary of $A_{n}$. Take the function $f$ on $M$ characterized by the following three formulas:

$$
f\left|\left(M-\bigcup_{n=1}^{\infty}\left(A_{n} \cup A_{n}^{\prime}\right)\right)=0, \quad f\right|\left(\bigcup_{n=1}^{\infty} A_{n}^{\prime}\right)=1,
$$

and for $x$ in $A_{n}(n=1,2, \cdots)$,

$$
f(x)= \begin{cases}1-\frac{|x|^{2-m}-a_{n}^{-1}}{\left(2 a_{n}\right)^{-1}-a_{n}^{-1}} & (m \geqq 3) \\ -a_{n} \log \frac{|x|}{a_{n}} & (m=2) .\end{cases}
$$

Clearly $f$ is bounded, continuous, and $A C L$ on $M$ and

$$
D_{M}(f)=\sum_{n=1}^{\infty} D_{A_{n}}(f) \leqq \tau^{1+m / 2} \sum_{n=1}^{\infty} \boldsymbol{D}_{A_{n}}(f) \text {. }
$$

On the other hand, we see by a simple calculation that

$$
\boldsymbol{D}_{A_{n}}(f)= \begin{cases}\Gamma(1+m / 2)^{-1} \pi^{m / 2}(m-2) a_{n} & (m \geqq 3) \\ 2 \pi a_{n} & (m=2) .\end{cases}
$$

In view of the choice of $a_{n}$, we conclude that $D_{M}(f)<\infty$.

Therefore $f \in \boldsymbol{R}(M)$ and a fortiori $f$ is continuous on $M^{*}$. In particular $f$ must be continuous at $p^{*}$. Take a point $q_{n}$ in the outer boundary of $A_{n}$ for each $n=1,2, \cdots$. Then since $f\left(q_{n}\right)=0$ and $\lim _{n \rightarrow \infty} q_{n}=p^{*}$, we would have $f\left(p^{*}\right)=0$. On the other hand, since $f\left(p_{n}\right)=1(n=1,2, \cdots)$ and $\lim _{n \rightarrow \infty} p_{n}=p^{*}$, we must have $f\left(p^{*}\right)=1$. 
This is a contradiction, and the proof of the Proposition is herewith complete.

22. Let $M_{1}$ and $M_{2}$ be Riemannian manifolds. An important rensequence of the preceding proposition is the following.

Proposition. Any homeomorphism $T^{*}$ of $M_{1}^{*}$ onto $M_{2}^{*}$ maps $M_{1}$ onto $M_{2}$, i.e. $T^{*}\left(M_{1}\right)=M_{2}$. The mapping $T=T^{*} \mid M_{1}$ is a homeomorphism of $M_{1}$ onto $M_{2}$.

In fact, let $p \in M_{1}$. Since $p$ is $G_{\delta}$ in $M_{1}, T^{*} p$ is also $G_{\delta}$ in $M_{2}^{*}$ and a fortiori $T^{*} p \in M_{2}$. Thus $T^{*}\left(M_{1}\right) \subset M_{2}$. By the symmetry, $T^{*}\left(M_{1}\right) \supset M_{2}$.

A homeomorphism $T$ of $M_{1}$ onto $M_{2}$ which can be continued to a homeomorphism of $M_{1}^{*}$ onto $M_{2}^{*}$ is said to be a Royden map. The above theorem shows that the Royden maps exhaust the homeomorphisms of $M_{1}^{*}$ onto $M_{2}^{*}$. For potential-theoretic properties and a characterization of Royden maps, we refer to Nakai [6].

23. We are ready to prove the following

THEOREM. Any algebraic isomorphism $\sigma$ of the Royden algebra $\boldsymbol{R}\left(M_{1}\right)$ onto $\boldsymbol{R}\left(M_{2}\right)$ induces and is induced by a Dirichlet mapping $T$ of $M_{1}$ onto $M_{2}$.

Let $T$ be a Dirichlet mapping of $M_{1}$ onto $M_{2}$. Then the mapping $\sigma$ defined by $f \rightarrow f^{\sigma}=f \circ T^{-1}$ is clearly an algebraic isomorphism of $\boldsymbol{R}\left(M_{1}\right)$ onto $\boldsymbol{R}\left(M_{2}\right)$.

Conversely, let $\sigma$ be an algebraic isomorphism of $\boldsymbol{R}\left(M_{1}\right)$ onto $\boldsymbol{R}\left(M_{2}\right)$. For a fixed $p^{*} \in M_{1}^{*}$, define a functional $T^{*}\left(p^{*}\right)$ on $\boldsymbol{R}\left(M_{2}\right)$ by

$$
\left(T^{*}\left(p^{*}\right)\right)(f)=f^{\tau}\left(p^{*}\right)
$$

for $f \in \boldsymbol{R}\left(M_{2}\right)$, where $\tau=\sigma^{-1}$. Clearly $T^{*}\left(p^{*}\right)$ is an algebraic homomorphism from $\boldsymbol{R}\left(M_{2}\right)$ onto the field of real numbers with $\left(T^{*}\left(p^{*}\right)\right)(1)=1$. Therefore $T^{*}\left(p^{*}\right) \in M_{2}^{*}$ and

$$
f\left(T^{*}\left(p^{*}\right)\right)=f^{\tau}\left(p^{*}\right)
$$

for every $f \in \boldsymbol{R}\left(M_{2}\right)$.

As a consequence of (38), we deduce that $T^{*}$ is a homeomorphism of $M_{1}^{*}$ onto $M_{2}^{*}$. By Proposition 22, $T^{*} \mid M_{1}=T$ is a homeomorphism of $M_{1}$ onto $M_{2}$ and (38) takes on the form $f \circ T=f^{\tau}$ for every $f \in \boldsymbol{R}\left(M_{2}\right)$. Therefore $f^{o}=f \circ T^{-1}$ for every $f \in \boldsymbol{R}\left(M_{1}\right)$. The proof is thus complete.

24. We are now in a position to prove our main result, Theorems 
4 and 5 (cf. $\S 1$ ). Let $T$ be a quasiconformal mapping of $M_{1}$ onto $M_{2}$ for $m=2$, and a quasi-isometry of $M_{1}$ onto $M_{2}$ for $m \geqq 3$. By Theorem 11 in $\S 3, T$ is a Dirichlet mapping of $M_{1}$ onto $M_{2}$. Therefore $T$ induces an algebraic isomorphism $f \rightarrow f^{\sigma}=f \circ T^{-1}$ of $\boldsymbol{R}\left(M_{1}\right)$ onto $\boldsymbol{R}\left(M_{2}\right)$.

Conversely, suppose an algebraic isomorphism $\sigma$ of $\boldsymbol{R}\left(M_{1}\right)$ onto $\boldsymbol{R}\left(M_{2}\right)$ is given. By the above theorem 23, $\sigma$ is induced by a Dirichlet mapping $T$ of $M_{1}$ onto $M_{2}$, i.c. $f^{\sigma}=f \circ T^{-1}$. Again by Theorem 11 in $\S 3, T$ is a quasiconformal mapping of $M_{1}$ onto $M_{2}$ for $m=2$, and a quasi-isometry of $M_{1}$ onto $M_{2}$ for $m \geqq 3$.

The proofs of Theorems 4 and 5 are herewith complete.

\section{REFERENCES}

1. F. Gehring, Quasiconformal mappings in space, Bull. Amer. Math. Soc., 69 (1963), 146-164.

2. H. Künzi, Quasikonforme Abbildungen, Springer-Verlag, 1960.

3. M. Nakai, Some topological properties on Royden's compactification of a Riemann surface, Proc. Japan Acad., 36 (1960), 555-559.

4. - Algebraic criterion on quasiconformal equivalence of Riemann surfaces, Nagoya Math. J., 16 (1960), 157-184.

5. — Continuity in mixed norms, Proc. Japan Acad., 45 (1969), 385-387.

6. - On parabolicity and Royden compactifications of Riemannian manifolds, Proc. International Congress Functional Analysis, 1969.

7. —, Radon-Nikodym densities and Jacobians, Pacific J. Math., (to appear).

8. M. Nakai-L. Sario, Classification and deformation of Riemannian spaces, Math. Scand., 20 (1967), 193-208.

9. C. Rickart, General Theory of Banach Algebras, Van Nostrand, 1960.

10. H. Rademacher, Über partielle und totale Differenzierbarkeit von Funktionen mehrerer Variabeln und Über die Transformation der Doppelintegrale, Math. Ann., 79 (1919), 340-359.

11. H. Royden, Harmonic functions on open Riemann surfaces, Trans. Amer. Math. Soc., 73 (1952) 40-94.

12. - On the ideal boundary of a Riemann surface, Contributions to the theory of Riemann surfaces, Princeton, 1953.

13. S. Saks, Theory of the Integral, Warszawa, 1937.

14. L. Sairo-M. Nakai, Classification Theory of Riemann Surfaces, Springer-Verlag, 1970.

15. M. Stepanoff, Sur les conditions de l'existence de la différentielle totale, Rec. Math. Soc. Math. Moscou, 32 (1925), 511-526.

16. M. Tsuji, Change of variables in the multiple Lebesgue integrals, J. Math. Soc. Japan, 2 (1950), 48-56.

17. K. Yoshida, Functional Analysis, Springer-Verlag, 1965.

Received September 1, 1970 and in revised form December 21, 1970. The work was sponsored by the U.S. Army research Office-Durham, Grant No. DA-AROD-31-124-G855, while the author was a Visiting Professor at the University of California, Los Angeles, in 1969 .

NAGOYA UNIVERSITY

AND

UNiversity of CALIFornia, Los ANGeles 


\title{
PACIFIC JOURNAL OF MATHEMATICS
}

\section{EDITORS}

\author{
H. SAMELSON \\ Stanford University \\ Stanford, California 94305 \\ C. R. HobBY \\ University of Washington \\ Seattle, Washington 98105
}

J. DugundJI

Department of Mathematics

University of Southern California

Los Angeles, California 90007

RICHARD ARENS

University of California

Los Angeles, California 90024

\section{ASSOCIATE EDITORS}
E. F. BECKENBACH
B. H. NeumanN
F. WOLF
K. YOSHIDA

\section{SUPPORTING INSTITUTIONS}

\author{
UNIVERSITY OF BRITISH COLUMBIA \\ CALIFORNIA INSTITUTE OF TECHNOLOGY \\ UNIVERSITY OF CALIFORNIA \\ MONTANA STATE UNIVERSITY \\ UNIVERSITY OF NEVADA \\ NEW MEXICO STATE UNIVERSITY \\ OREGON STATE UNIVERSITY \\ UNIVERSITY OF OREGON \\ OSAKA UNIVERSITY
}

\author{
UNIVERSITY OF SOUTHERN CALIFORNIA \\ STANFORD UNIVERSITY \\ UNIVERSITY OF TOKYO \\ UNIVERSITY OF UTAH \\ WASHINGTON STATE UNIVERSITY \\ UNIVERSITY OF WASHINGTON
AMERICAN MATHEMATICAL SOCIETY
NAVAL WEAPONS CENTER

The Supporting Institutions listed above contribute to the cost of publication of this Journal, but they are not owners or publishers and have no responsibility for its content or policies.

Mathematical papers intended for publication in the Pacific Journal of Mathematics should be in typed form or offset-reproduced, (not dittoed), double spaced with large margins. Underline Greek letters in red, German in green, and script in blue. The first paragraph or two must be capable of being used separately as a synopsis of the entire paper. The editorial "we" must not be used in the synopsis, and items of the bibliography should not be cited there unless absolutely necessary, in which case they must be identified by author and Journal, rather than by item number. Manuscripts, in duplicate if possible, may be sent to any one of the four editors. Please classify according to the scheme of Math. Rev. Index to Vol. 39. All other communications to the editors should be addressed to the managing editor, Richard Arens, University of California, Los Angeles, California, 90024.

50 reprints are provided free for each article; additional copies may be obtained at cost in multiples of 50 .

The Pacific Journal of Mathematics is published monthly. Effective with Volume 16 the price per volume (3 numbers) is $\$ 8.00$; single issues, $\$ 3.00$. Special price for current issues to individual faculty members of supporting institutions and to individual members of the American Mathematical Society: $\$ 4.00$ per volume; single issues $\$ 1.50$. Back numbers are available.

Subscriptions, orders for back numbers, and changes of address should be sent to Pacific Journal of Mathematics, 103 Highland Boulevard, Berkeley, California, 94708.

\section{PUBLISHED BY PACIFIC JOURNAL OF MATHEMATICS, A NON-PROFIT CORPORATION}

Printed at Kokusai Bunken Insatsusha (International Academic Printing Co., Ltd.), 270, 3chome Totsuka-cho, Shinjuku-ku, Tokyo 160, Japan. 


\section{Pacific Journal of Mathematics}

\section{Vol. 40, No. $2 \quad$ October, 1972}

Louis I. Alpert and L. V. Toralballa, An elementary definition of surface area in $E^{n+1}$ for smooth surfaces...........................

Eamon Boyd Barrett, A three point condition for surfaces of constant mean curvature........................................

Jan-Erik Björk, On the spectral radius formula in Banach algebras ....... 279

Peter Botta, Matrix inequalities and kernels of linear transformations . . . . 285

Bennett Eisenberg, Baxter's theorem and Varberg's conjecture ........... 291

Heinrich W. Guggenheimer, Approximation of curves .............. 301

A. Hedayat, An algebraic property of the totally symmetric loops associated with Kirkman-Steiner triple systems ....................... 305

Richard Howard Herman and Michael Charles Reed, Covariant representations of infinite tensor product algebras ................

Domingo Antonio Herrero, Analytic continuation of inner

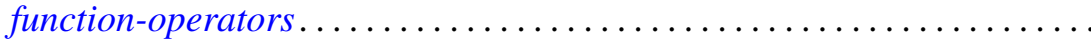

Franklin Lowenthal, Uniform finite generation of the affine group......... 341

Stephen H. McCleary, 0-primitive ordered permutation groups .......... 349

Malcolm Jay Sherman, Disjoint maximal invariant subspaces .......... 373

Mitsuru Nakai, Radon-Nikodým densities and Jacobians .............. 375

Mitsuru Nakai, Royden algebras and quasi-isometries of Riemannian manifolds. . .

Russell Daniel Rupp, Jr., A new type of variational theory sufficiency

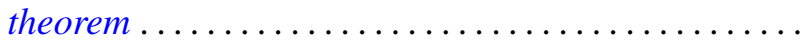

Helga Schirmer, Fixed point and coincidence sets of biconnected multifunctions on trees..........................

Murray Silver, On extremal figures admissible relative to rectangular

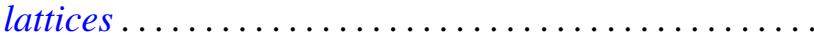

James DeWitt Stein, The open mapping theorem for spaces with unique segments ...

Arne Stray, Approximation and interpolation

Donald Curtis Taylor, A general Phillips theorem for $C^{*}$-algebras and some applications

Florian Vasilescu, On the operator $M(Y)=T Y S^{-1}$ in locally convex algebras...

Philip William Walker, Asymptotics for a class of weighted eigenvalue problems...

Kenneth S. Williams, Exponential sums over $\mathrm{GF}\left(2^{n}\right)$. 